\title{
PEG-fDAO Reduces Lung Inflammation in Chronic Granulomatous Disease Mice Post Challenge With Nonviable Candida Albicans
}

\section{Hiroyuki Nunoi ( $\square$ hnunoi@aisenkai-nichinan.jp )}

Aisenkai Nichinan Hospital https://orcid.org/0000-0003-3067-0809

\section{Peiyu Xie}

Faculty of Pharmaceutical Sciences, Sojo University

\section{Hideaki Nakamura}

Sojo University Faculty of Pharmaceutical Sciences Graduate School of Pharmaceutical Sciences: Sojo Daigaku Yakugakubu Daigakuin Yakugaku Kenkyuka

\section{Yasuaki Aratani}

Yokohama City University School of Medicine Graduate School of Medicine: Yokohama Shiritsu Daigaku Igakubu Daigakuin Igaku Kenkyuka

\section{Jun Fang}

Sojo University Faculty of Pharmaceutical Sciences Graduate School of Pharmaceutical Sciences: Sojo Daigaku Yakugakubu Daigakuin Yakugaku Kenkyuka

\section{Toyoki Nishimura}

University of Miyazaki Faculty of Medicine Graduate School of Medicine and Nursing: Miyazaki Daigaku Igakubu Daigakuin Ikagaku Kangogaku Kenkyuka

Hiroaki Kataoka

University of Miyazaki Faculty of Medicine Graduate School of Medicine and Nursing: Miyazaki Daigaku Igakubu Daigakuin Ikagaku Kangogaku Kenkyuka

Hiroshi Maeda

BioDynamics Research Foundation

\section{Makoto Matsukura}

Sojo University: Sojo Daigaku

\section{Research Article}

Keywords: nCA-induced lung inflammation, CGD mice, PEG-D-amino acid oxidase (PEG-fDAO), enzyme replacement therapy

Posted Date: August 20th, 2021

DOI: https://doi.org/10.21203/rs.3.rs-805346/v1

License: (c) (i) This work is licensed under a Creative Commons Attribution 4.0 International License. Read Full License 


\section{Abstract}

We previously reported that polyethylene glycol-conjugated recombinant porcine D-amino acid oxidase (PEG-pDAO) could supply reactive oxygen species (ROS) to defective NADPH oxidase in neutrophils of patients with chronic granulomatous disease (CGD), and neutrophils regain bactericidal activity in vitro. In the present study, we employed an in vivo nonviable Candida albicans (nCA)-induced lung inflammation model using gp91-phox knockout CGD mice and novel PEG conjugates of Fusarium spp. D-amino acid oxidase (PEG-fDAO), rather than PEG-pDAO. Using three experimentation strategies with the in vivo lung inflammation model, the mouse body weight, lung weight, and lung pathology were evaluated to confirm the efficacy of ROS-generating enzyme replacement therapy with PEG-fDAO. The lung weight and pathological findings were significantly ameliorated by the administration of PEG-fDAO followed by intraperitoneal injection of D-phenylalanine or D-proline. These data suggest that PEG- fDAO with the function of targeted delivery to the nCA-induced inflammation site is applicable in the treatment of inflammation in CGD in vivo.

\section{Introduction}

Chronic granulomatous disease (CGD) is a primary immunodeficiency characterized by the inability of phagocytes to produce reactive oxygen species (ROS) due to a defect in the NADPH oxidase complex. Clinically, CGD patients frequently suffer from bacterial infections and excessive inflammatory disorders, such as CGD-associated bowel inflammation and sterile granulomas. ROS generation is crucial for the phagocytic killing of ingested microbes [1] and inflammasome signaling processes, such as the Caspase-1 activation platform for cytokine production [2-4], the Keap1-Nrf2 pathway for antioxidative stress [5], and efferocytosis through phosphatidylserine and its receptor [6].

Anti-inflammatory cytokine therapies (anti-interleukin (IL)-1 antibody, etc.) have been proven to alleviate excessive cytokine production in an in vivo CGD mouse model and clinical studies on severe

colitis [4]. In addition, a PPARy agonist (pioglitazone) has been confirmed to enhance mitochondrial ROS production and partially restore host defense in a CGD mouse model [6]. Bone marrow transplantation or gene therapy with a lentiviral vector and myeloid-specific promoter has been proposed as a successful curative therapy [7]. Although bone marrow transplantation and gene therapy are thought to be theoretically curative treatments, residual pathogenic components, live or dead, lead to severe vital reactions, such as inflammation and/or organic damage, in CGD patients. Unprecedented antibiotic treatments are pathogen-specific remedies for the removal of pathogens. Potent pathogen-specific antibiotic treatments can kill pathogens but may not prevent the excessive inflammation that can persist due to the presence of residual materials of killed pathogens, such as fungal cell wall bglucan. Moreover, persistent chronic inflammation in patients with CGD also leads to impairment of hematopoietic stem cell functions [8].

To overcome these limitations, we aimed to develop a novel enzyme replacement therapy with D-amino acid oxidase (DAO) for supplying $\mathrm{H} 2 \mathrm{O} 2$ in vivo. DAO is a flavo-enzyme that selectively catalyzes the oxidative deamination of $\mathrm{D}$-amino acids, leading to the generation of the corresponding imino acid and $\mathrm{H} 2 \mathrm{O} 2$. We previously reported that DAO restores the bactericidal activity of ROS-deficient neutrophils by supplying $\mathrm{H} 2 \mathrm{O} 2$ in vitro [9]. The advantages of using fungal DAO are easy availability of this enzyme and more potent selective activity compared with that of mouse or human DAO. [10]. This phenomenon is called the enhanced permeability and retention effect [11]. 
In this study, a novel polyethylene glycol-conjugated Fusarium spp. DAO (PEG-fDAO) was characterized and evaluated for its in vivo anti-inflammatory activity in a lung inflammation model induced with nonviable Candida albicans (nCA) using gp91-phox knockout CGD mice. Lack of ROS production has been shown to result in high levels of proinflammatory mediators (IL-1b, tumor necrosis factor-a, and keratinocyte chemoattractant) through the inflammasome activation system (dectin-1 receptor) in neutrophils and macrophages [3]. In this model, viable fungi are not always a prerequisite for the development of inflammation in CGD mice [12].

Therefore, using this model, we propose that oxidase replacement therapy with the novel PEG-fDAO could be applicable in the treatment of inflammation in vivo.

\section{Materials And Methods}

\section{Reagents}

DAO isolated from Fusarium spp. (fDAO) was a kind gift from lkeda Food Research Co., Ltd, Minami- ku Kumamoto, Japan. MTT (3-(4,5-dimethylthiazol-2-yl)-2,5-diphenyltetrazolium bromide) and H2DCFDA (2',7'-

dichlorodihydrofluorescein diacetate) were obtained from Sigma-Aldrich (St. Louis, MO, USA) and Molecular Probes (Eugene, OR, USA), respectively. $\mathrm{H} 2 \mathrm{O} 2$ and tert-butyl hydroperoxide (t-BuOOH) were purchased from Wako Pure Chemical Industries, Ltd. (Osaka, Japan). D-Phenylalanine, D-proline, and rhodamine B isothiocyanate were purchased from Wako Pure Chemical Industries, Ltd.

Succinimidyl-activated PEG (SUNBRIGHT MEGC-50HS-PEG), with an average molecular size of 5250 Da, was purchased from NOF Corp., Tokyo, Japan. All other reagents were of reagent grade and used without further purification.

\section{Animals}

CGD mice (gp91-phox knockout) obtained from Dr. Mary C. Dinauer [13] were backcrossed at least 12 times with C57BL/ 6 mice to ensure similar genetic backgrounds. Experiments on 8-12-week-old CGD C57BL/6 mice were performed according to the guidelines of the Laboratory Protocol of Animal Handling, Sojo University Faculty of Pharmaceutical Sciences. All animals were housed under specific pathogen-free conditions.

\section{Preparation of nCA}

nCA was kindly gifted by Dr. Ohno [14]. Briefly, a C-limiting medium was used to grow Candida albicans and cultured at $27^{\circ} \mathrm{C}$ with aeration. Viable cells were collected by centrifugation, killed with ethanol, and dried with acetone. The resulting nCA was suspended in phosphate-buffered saline (PBS).

\section{Preparation of PEG-fDAO}

PEGylation of fDAO was conducted as described previously [15]. In brief, to the fDAO solution (2.0 $\mathrm{mg} / \mathrm{mL}$ protein in $0.1 \mathrm{M}$ sodium bicarbonate), succinimide-activated PEG was added at a 3-fold molar excess of PEG to free the amino groups in fDAO and allowed to react for 1 hour at $4{ }^{\circ} \mathrm{C}$. The reaction mixture containing PEG-fDAO was purified to remove free PEG and other low-molecular-weight reactants by ultrafiltration with a YM-10 membrane 


\section{Evaluation of the enzymatic activities of fDAO and PEG- fDA}

The enzymatic activity of fDAO and PEG-fDAO were determined by a horseradish peroxidase-coupled colorimetric assay with o-dianisidine as the substrate. In this assay, the substrate was reduced, and a color developed with a maximal absorption of $460 \mathrm{~nm}$. D-alanine was used as the substrate for fDAO at a final concentration of $10 \mathrm{mM}$. The enzymatic reaction was performed at $25^{\circ} \mathrm{C}$ in $0.1 \mathrm{M}$ Tris- $\mathrm{HCl}$ buffer $(\mathrm{pH} \mathrm{8.2)}$, where $1 \mathrm{U}$ of fDAO activity is defined as the rate of formation of $1 \mu \mathrm{mol}$ of $\mathrm{H} 2 \mathrm{O} 2$ per minute. The maximal rate of activity (Vmax) and $\mathrm{Km}$ for each D-amino acid were calculated by curve fitting of the non- linear plot of reaction rate versus substrate concentration using the gnu plot software.

\section{Analysis of in vivo pharmacokinetics of fDAO and PEG- fDAO using the plasma}

For the in vivo pharmacokinetics analysis, fDAO or PEG-fDAO $(20 \mathrm{U} / \mathrm{mL}, 0.1 \mathrm{~mL} / \mathrm{mouse}, \mathrm{n}=3)$ was injected intravenously into C57BL/6 (BALB/CAjcl) mice. Blood was withdrawn from the medial canthus of the eye using a microhematocrit at $0.5,4,24,48$, and 72 hours after fDAO or PEG-fDAO administration. Each blood sample was centrifuged, and the plasma was obtained in an ice-cold buffer (100 mM Tris- $\mathrm{HCl}, \mathrm{pH} 8.0)$ containing a mixture of protease inhibitors ( $1 \mathrm{mM}$ PMSF, $10 \mu \mathrm{g} / \mathrm{mL}$ leupeptin, and $2.5 \mathrm{mM}$ EDTA). The DAO activity in the plasma was determined based on the formation of pyruvic acid during the reaction between D-Alanine and DAO, as described previously [15].

\section{Induction of lung inflammation by nCA aspiration}

Mice were anesthetized by intraperitoneal injection with $200 \mathrm{mg} / \mathrm{kg}$ of 2,2,2-tribromoethanol (Sigma- Aldrich), as described by Dr. Aratani. CGD mice were challenged with intranasal administration of $10^{7} \mathrm{nCA}$ cells in a volume of $30 \mu \mathrm{L}$ of PBS, and control mice were administered $30 \mu \mathrm{L}$ of PBS alone [12]. Since we had no information on the time course of in vivo production of $\mathrm{H} 2 \mathrm{O} 2$ induced by PEG- fDAO with D-amino acids in the nCA-induced lung inflammation model, a short, medium, and long treatment schedule was explored.

\section{Experiment-1 (Exp-1)}

Mice were divided into two groups. The first group was administered nCA intranasally as the positive control $(n=5)$. The second group was administered nCA intranasally on day 0 of experiment and PEG- fDAO was injected through the tail vein on the second day, followed by intraperitoneal administration of D-phenylalanine $(0.1 \mathrm{M}, 0.5$ $\mathrm{mL} /$ mouse) on the third, fourth, and fifth days $(n=5)$. Mice were sacrificed on the eighth day. 


\section{Experiment-2 (Exp-2)}

Mice were divided into three groups. The first group was administered PBS intranasally as the negative control ( $\mathrm{n}=$ $3)$. The second group was administered $n C A$ intranasally as the positive control $(n=3)$. The third group was administered $\mathrm{nCA}$ on day 0 and injected with PEG-fDAO $(10 \mathrm{U} / \mathrm{mL}, 0.1 \mathrm{~mL} / \mathrm{mouse})(\mathrm{n}=3)$ through the tail vein on the fourth day, followed by intraperitoneal injection of D-phenylalanine $(0.1 \mathrm{M}, 0.5 \mathrm{~mL} / \mathrm{mouse})$ on the fifth, sixth, and seventh days instead of D-proline. Rhodamine-labeled bovine serum albumin (rhodamine-BSA) (10 mg/kg) was injected one day before sacrifice on the $14^{\text {th }}$ day. Fluorescent imaging of the excised lungs was conducted using the IVIS Lumina XR (excitation: 555-585 emission: 695-770 nm; PerkinElmer Japan Co., Ltd., kanagawa, Japan).

\section{Experiment-3 (Exp-3)}

Mice were divided into three groups. The first group was administered PBS intranasally as the negative control ( $\mathrm{n}=$ $3)$. The second group was administered nCA intranasally as the positive control $(n=3)$. The third group was administered $\mathrm{nCA}$ on day 0 and injected with PEG-fDAO $(10 \mathrm{U} / \mathrm{mL}, 0.1 \mathrm{~mL} / \mathrm{mouse})(\mathrm{n}=3)$ on the ninth day, followed by intraperitoneal injection of D-proline $\left(1 \mathrm{M}, 0.5 \mathrm{~mL} /\right.$ mouse) on the $10^{\text {th }}, 11^{\text {th }}$, and $12^{\text {th }}$ days. Rhodamine-BSA (10 $\mathrm{mg} / \mathrm{kg}$ ) was injected one day before sacrifice on the $21^{\text {st }}$ day. Fluorescent imaging of the excised lungs was performed using the IVIS Lumina XR (PerkinElmer Japan Co., Ltd.).

\section{Analysis of the lung pathology}

Mice were sacrificed on the eighth day in Exp-1, $14^{\text {th }}$ day in Exp-2, or $21^{\text {st }}$ day in Exp-3 after nCA administration. The lungs were removed and fixed in $10 \%$ buffered formalin. For light microscopy, tissues were fixed overnight, dehydrated in graded ethanol solutions, embedded in paraffin, sectioned at 2- $\mu \mathrm{m}$ thickness, and stained with hematoxylin and eosin using standard protocols.

\section{Statistical analysis}

Statistical analysis of the obtained data was performed using a two-tailed unpaired Student's $t$-test. Differences were considered statistically significant at $p<0.05$.

\section{Results}

\section{Physicochemical characterization of fDAO and PEG-fDAO}

Characterization of PEG-fDAO is summarized as follows. The reaction between fDAO and succinimide-PEG resulted in five chain numbers of PEG conjugates on $\mathrm{FDAO}$, which was determined by quantification of the primary amine. The fDAO formed a tetramer $(165 \mathrm{kDa})$ in the physiological

solution, and the molecular weight of PEG-fDAO was calculated to be $206 \mathrm{kDa}$. An increase in the molecular size of fDAO by PEGylation was also confirmed by SDS-PAGE and size exclusion chromatography (data not shown). Enzyme-specific activity of fDAO was $26 \pm 1.3$ units/mg protein, and its enzyme activity was not altered by PEGylation (PEG-fDAO: $26 \pm 0.53$ units/mg protein). The Vmax and Km values of fDAO for each D-type amino acid 
were almost similar with those of PEG-fDAO. Vmax and $\mathrm{Km}$ values of fDAO and PEG-fDAO towards neutral D-amino acids also showed similar trends at the optimal $\mathrm{pH}$ of 8.2, and an inflammatory environmental $\mathrm{pH}$ of 6.5 (Supplemental Table 1). The enzymatic activity of PEG-fDAO at pH 6.5 was lower than that at pH 8.2, for all substrates tested. Similar substrate specificity was observed at pH 6.5 and 8.2.

D-phenylalanine showed the highest Vmax (Vmax $=18.6 \mu \mathrm{mol} \mathrm{H} 2 \mathrm{O} 2 / \mathrm{min}$ at $\mathrm{pH}$ 6.5) and was active even at low substrate concentrations $(\mathrm{Km}=0.22 \mathrm{mM}$ at pH 6.5) among the $\mathrm{D}$-amino acids tested. We employed D-proline in Exp-3 to compare the in vitro bactericidal activity ${ }^{1}$ with the in vivo anti-inflammatory activity $(\mathrm{Vmax}=6.4, \mathrm{Km}=5.2$ at pH 6.5). In Exp-1 and Exp-2, D-phenylalanine was selected as the substrate to confirm PEG-fDAO activity in vivo.

\section{In vivo pharmacokinetics of fDAO and PEG-fDAO using the plasma}

We employed a methodology to exclude denatured DAO proteins with lost enzymatic activity for measuring the enzymatic activity of DAO in vivo as in Figure 1 . The apparent half-life (t1/2) of fDAO $(n=3)$ and PEG-fDAO (n=7) are statistically $7.69 \pm 1.36$, hour, $33.68 \pm 3.10$ hour, respectively, and T-Test with un-paired samples shows that pvalue is 0.000000415 . Since the $1 / 2$ of PEG-fDAO appeared to be long enough, we employed intraperitoneal injection of D-amino acid on three consecutive days post PEG- fDAO administration.

\section{In vivo experimental findings}

In Exp-1 with an 8-day schedule, there was a marked mean body weight loss in the nCA group (-6.18 $\pm 2.28 \mathrm{~g})$ compared with that in the PEG-fDAO experimental group $(-4.14 \pm 2.74 \mathrm{~g})$, but the difference was not significant $(p=$ $0.236, n=5)$ (Figure $2 A$ and $2 B)$. The mean lung weight in the nCA group $(0.562 \pm 0.04 \mathrm{~g})$ was significantly higher than that in the PEG-fDAO experimental group $(0.314 \pm 0.095 \mathrm{~g})(p=0.001, n=5)$ (Figure $3 A)$. Images of the excised lungs are shown in Supplemental Figure 1. Rhodamine- labeled fluorescent imaging was not performed in this experiment.

In Exp-2 with a 14-day schedule and D-phenylalanine substrate, the mean body weight loss in the nCA group (-1.6 \pm $0.7 \mathrm{~g})$ was lower than that in the PEG-fDAO group $(-2.5 \pm 2.8 \mathrm{~g})(\mathrm{p}=0.618, \mathrm{n}=3)$ (Figure 2D and 2E). The mean lung weight in the nCA group $(0.283 \pm 0.025 \mathrm{~g})$ was significantly higher than that

in the PEG-fDAO group $(0.236 \pm 0.005 \mathrm{~g})(\mathrm{p}=0.035, \mathrm{n}=3)$ (Figure 3B 2/3). Furthermore, the mean lung weight in the control group was $0.24 \pm 0.01 \mathrm{~g}$, which was almost the same as that in the PEG-fDAO group $(p=0.64, n=3)$ (Figure 3B 1/3). To determine the inflammatory region in the lung, rhodamine- BSA was intravenously injected, and accumulated rhodamine-BSA in the lung was quantified by measuring the fluorescence intensity. Rhodaminelabeled fluorescence intensity in the excised lungs of mice from the nCA group was brighter and the tissue was more swollen than that observed for the mice in the PEG-fDAO and control groups (Figure 4A). The pathological findings were different in the nCA group; pneumonia and focal accumulation of neutrophils reacting to and phagocytizing eosinophilic filaments were observed in the nCA group (Figure 5C/D), but these were milder in the lung tissues from mice in the PEG-fDAO group (Figure 5E/F).

In Exp-3 with a 21-day schedule, there was no marked difference in the mean body weight loss in the nCA group $(-0.33 \pm 0.68 \mathrm{~g})$ compared with that in the PEG-fDAO group $(-0.17 \pm 0.87 \mathrm{~g})(\mathrm{p}=0.66, \mathrm{n}=3)$ (Figure $2 \mathrm{G}$ and $2 \mathrm{~F})$. The mean lung weight in the nCA group $(0.334 \pm 0.065 \mathrm{~g})$ was heavier than that in the PEG-fDAO group $(0.230 \pm 0.021$ 
$g)$, but the difference was not significant $(p=0.058, n=3)$ (Figure $3 C$ ). In the control group, the mean lung weight was $0.156 \pm 0.020 \mathrm{~g}$. Rhodamine-labeled fluorescence intensity in the excised lungs from mice in the nCA group was brighter and the tissue was more swollen than that noted for mice in the PEG-fDAO and control groups (Figure 4B). The pathological findings were particularly different in the nCA group, showing pneumonia with accumulation of eosinophilic filament-phagocytizing neutrophils, accompanied by granulomatous accumulation of histiocytic cells and lymphoid cells. These pathological changes were apparently mild, with the eosinophilic filaments fragmented and smaller in the tissues from mice in the PEG-fDAO group (Figure 5K/L) compared with that found in mice from the nCA group (Figure $5 \mathrm{l} / \mathrm{J}$ ). Unexpectedly, a small amount of granuloma formation with giant cells was observed in the PEG-fDAO group.

\section{Discussion}

In the present study, our pharmacokinetics analysis revealed that fDAO (165 kDa) was rapidly cleared from the circulation after intravenous infusion ( $\mathrm{t} 1 / 2=7.67 \pm 1.36$ hour $(\mathrm{n}=3)$ ), but PEG-fDAO $(206 \mathrm{kDa})$ was rather stable $(\mathrm{t} 1 / 2=33.68 \pm 3.10$ hour $(\mathrm{n}=7))$ (Figure 1$)$. Based on these findings, we employed PEG-fDAO administration, followed by administration of D-phenylalanine or D-proline for three days in the nCA-induced lung inflammation model to evaluate the anti-inflammatory effect of PEG-fDAO. According to studies on Aspergillus hyphae- [16] and sterile nCA- [12] induced lung inflammation models, sterile fungal cell wall (branched $(\beta-1,3)(\beta-1,6)$ glucan)-induced inflammation model with gp $91^{\%-}$ CGD mice [17] and zymosan (the same fungal cell wall-derived product)-induced lung inflammation model with $\mathrm{p} 47^{-/-}[18]$ and $\mathrm{p} 91^{\%-}[19]$ CGD mice, the MAPK signaling pathway (ERK1/2 and NF$\mathrm{KB}$ ) involving toll-like receptor 2, dectin-1 receptor and cytokines (IL-1b, tumor necrosis factor-a, IL-17, keratinocyte chemoattractant, and granulocyte colony-stimulating factor) and leukotriene B4 [19] are activated on the second day or earlier post stimulation. The net balance of cytokine and leukotriene B4 expression in the lungs of CGD mice in response to the fungal cell wall component is prone to be in a proinflammatory state, which could promote a continued inflammatory response $[16,19]$. Based on these previous findings, we administered PEG-fDAO on the second day, followed by an intraperitoneal injection of D-phenylalanine for three days in Exp-1. Endo et al [12] reported that nCA-treated CGD mice show prominent airway accumulation of inflammatory cells at 3 and 6 days post nCA administration.

Similarly, the nCA-treated CGD mice in Exp-1 showed a prominent loss of body weight and gain of the lung weight. In contrast, nCA-treated CGD mice injected with PEG-fDAO and D-phenylalanine showed a significantly lower loss of body weight and gain of the lung weight (Figures 2A/B and Figure $3 \mathrm{~A}$ ) compared with that observed in the control group. These differences were also apparent from a physiological point of view.

Next, to determine the effect of PEG-fDAO over a longer period, as in the Aspergillus hyphae-induced lung inflammation model [16], PEG-fDAO was administered on the fourth day in Exp-2 with a 14-day schedule, and the ninth day in Exp-3 with a 21-day schedule. We found the body and lung weight to tend to recover over the experimental time course. In Exp-2, the loss of body weight in the nCA group was approximately the same as that in the PEG-fDAO group $(p=0.62)$ (Figure 2D/E), but the lung weight gain in the nCA group was significantly higher $(p=$ 0.035 ) than that in the PEG-fDAO group (Figure 3B). Furthermore, the lung pathology in the nCA group appeared to be stronger than that in the PEG- fDAO group (Figure 5C/D vs 5E/F). In Exp-3, the loss of body weight in the nCA group was higher than that in the PEG-fDAO group, but the difference was not significant $(p=0.66)($ Figure $2 G / H)$. The lung weight gain in the nCA group was not significantly high $(p=0.058)$, but it was heavier than that in the PEG-fDAO group (Figure 3C; 2 vs 3). Although PEG-fDAO seemed effective in ameliorating the physiological changes induced by nCA stimulation, we could not confirm if this effect was significant through these evaluations. 
Consistent with a previous study by Dinauer [16], which had a 21-day experimental schedule with intratracheal administration of Aspergillus fumigatus hyphae to $\mathrm{gp} 91^{/-}$mice, many granulomatous formations in our Exp-3 were observed in the pathological lung specimens from mice in the nCA group (Figure $5 \mathrm{l} / \mathrm{J}$ ) but a few in the those from mice in the PEG-fDAO group (Figure 5K/L). Granulomatous formations were not apparent in Exp-2, but a few macrophage accumulations around the eosinophilic Candida body were found in the pathological lung specimens from mice in the nCA group (Figure 5C/D and not in those from mice in the PEG-fDAO group (Figure 5E/F). In addition, the lung pathology in the nCA group (Figure $5 \mathrm{I} / \mathrm{J}$ ) was stronger than that observed in the PEG-fDAO group in Exp-3(Figure 5K/L). These pathological findings also support the idea that PEG-fDAO administration, followed by D-amino acid treatment, would ameliorate the pathological changes induced by nCA stimulation.

According to the characterization of PEG-fDAO, the Km value of D-phenylalanine was approximately 24-40 times lower than that of D-proline, but the blood concentration of D-phenylalanine or D-proline was supposed to be 2-3 times higher than the Km value of both D-amino acids. In addition, the Vmax of PEG-fDAO for D-phenylalanine was approximately three times higher than that for D-proline (Supplemental Table 1). Since D-phenylalanine has lower solubility than D-proline in aqueous media, bolus injectable D-phenylalanine was 10-fold lower than D-proline (Dphenylalanine: $0.1 \mathrm{M}, 0.5 \mathrm{~mL} /$ mouse (Exp-1, 2) and D-proline: $1 \mathrm{M}, 0.5 \mathrm{~mL} / \mathrm{mouse}$ (Exp-3). Therefore, although PEGfDAO activity was higher towards D-phenyalanine than D-proline, a low dose of D-phenylalanine might result in a therapeutic efficacy comparable to that of D-proline in alleviating lung inflammation. However, more stable conditions and protocols are necessary to confirm the apparent effects of PEG-fDAO observed in this model and develop clinical applications.

Therefore, in these series of experiments, PEG-fDAO administration followed by D-amino acid injection was apparently useful for the treatment of nCA-induced lung inflammation, especially in Exp-1, but also in Exp-2 and Exp-3. Considering the mechanism of the nCA-induced pneumonia model, $\mathrm{H} 2 \mathrm{O} 2$ seems to be involved in many inflammatory processes. As Endo et al [12] reported in their 3-day pneumonia model, a low number of MAPKs in CGD mice may lead to prolonged phosphorylation of ERK1/2. Segal et al [18] showed impaired Nrf2 activity and increased NF-KB activation in zymosan- treated mononuclear cells from X-linked CGD patients. Furthermore, many cytokines are thought to be involved in granuloma formation with giant cells [20] wherein $\mathrm{H} 2 \mathrm{O} 2$ is involved. As neutrophil-derived ROS, the targeted delivery of PEG-fDAO could also restore the anti-inflammatory responses by supplying $\mathrm{H} 2 \mathrm{O} 2$ to the site of inflammation. Setoguchi et al [21] also reported the therapeutic value of pulmonary granuloma by controlling nitric oxide and ROS.

In conclusion, we prepared PEG-fDAO, characterized its enzymatic properties, and evaluated its anti- inflammatory effect on gp91-phox knockout CGD mice. The features of PEG-fDAO were comparable to those of pDAO in terms of Vmax and substrate specificity, but PEG-fDAO was more stable than PEG- pDAO. Moreover, PEG-fDAO exhibited anti-inflammatory effects in CGD mice with nCA-induced lung inflammation. Nonetheless, a protocol with a more optimized regimen will be necessary to confirm the anti-inflammatory effects of PEG-fDAO.

\section{Declarations}

\section{Acknowledgements}

The authors thank Mary C. Dinauer, Indiana University School of Medicine, for kindly providing CGD mice. We also thank the laboratory students at Sojo University (Takahiro Hattori, Yuhki Funatsu, Kazuhiro Minowa, Arisa Nakahara, Kazumi Matsuura, Saki Ejima, and Kanako Ohhira) for their helpful contribution under the guidance of 
Makoto Matsukura. We would like to thank Editage (www.editage.com) for English language editing. This work was supported by JSPS KAKENHI (grant JP16K09972).

\section{Funding}

This work was supported by JSPS KAKENHI (grant JP16K09972).

\section{Conflicts of interest/Competing interests}

None of the authors has any conflicts of interest or any financial ties to disclose.

\section{Availability of data and material}

For original data, please contact hnunoi@med.miyazaki-u.ac.jp.

\section{Code availability}

Manuscript was written by MacBook Pro (13-inch,2016) with Microsoft R word for Mac (version 16.51), and Microsoft power point for Mac (version 16.51).

\section{Authors' contributions}

Hiroshi Maeda proposed basic concept of PEGylated DAO for the treatment of CGD. Makoto Matsukura and Hiroyuki Nunoi design the research. Hideaki Nakamura prepared materials especially PEG-fDAO. Yasuaki Aratani performed the first experiment with the nCA-induced lung inflammation model. Peiyu Xie, Jun Fang and collaborators performed the other experiments. Peiyu Xie did equal contribution for this work with Hiroyuki Nunoi. Analysis of in vivo pharmacokinetics of fDAO and PEG- fDAO using the plasma were performed by Makoto Matsukura and his coworkers. Hiroaki Kataoka conducted pathological evaluation. Hiroyuki Nunoi, Taiki Nisimura and Makoto Matsukura analyzed the data and wrote the first draft of the manuscript. All authors commented on the manuscript and approved it.

\section{Ethics approval}

This study was approved by the Ethics Review Board in Sojo University Faculty of Pharmaceutical Sciences (Permission number: 2017-P-027).

\section{Consent to participate}

This study was performed according to the guidelines of the Laboratory Protocol of Animal Handling, Sojo University Faculty of Pharmaceutical Sciences. All animals were housed under specific pathogen-free conditions.

\section{Consent for publication}

All authors have approved the manuscript and agree with submission.

\section{References}

1. Holland, S. M. 2013. Chronic granulomatous disease. Hematology/Oncology Clinics of North America 27: 8999. DOI:10.1016/j.hoc.2012.11.002. 
2. Luigi Franchi, Tatjana, and Eigenbrod, Raúl Muñoz-Planillo, and Gabriel Nuñez. 2009. The inflammasome: a Caspase- 1 activation platform regulating immune responses and disease pathogenesis. Nature Immunology 10: 241-247. DOI: 10.1038/ni.1703.

3. Felix Meissner, Reinhard A., Despina Seger, Alain Moshous, Janine Fischer, Reichenbach, and Arturo Zychlinsky. 2010. Inflammasome activation in NADPH oxidase defective mononuclear phagocytes from patients with chronic granulomatous disease. Blood 116: 1570-1573. DOI:10.1182/blood-2010-01-264218.

4. David, I., Brown, and Kathy K. Griendling. 2009. Nox proteins in signal transduction. Free Radical Biology \& Medicine 47: 1239-1253. DOI:10.1016/j.freeradbiomed.2009.07.023.

5. Stjepana Kovac, Plamena R., Kira M. Angelova, Ying Holmström, T. Zhang, Albena, Dinkova-Kostova, and Y. Andrey, and Abramov. 2015. Nrf2 regulates ROS production by mitochondria and NADPH oxidase. Biochimica et Biophysica Acta 1850: 794-801. DOI:10.1016/j.bbagen.2014.11.021.

6. Ruby, F., S. Fernandez-Boyanapalli, Stacey M. Courtney Frasch, Kenneth C. Thomas, Michael Malcolm, Ronald J. Nicks, Claudia V. Harbeck, Raphael Jakubzick, Peter M. Nemenoff, Steven M. Henson, Holland, and Donna L. Bratton. 2015. Pioglitazone restores phagocyte mitochondrial oxidants and bactericidal capacity in chronic granulomatous disease. Journal of Allergy and Clinical Immunology 135: 517-527. DOI:10.1016/j.jaci.2014.10.034.

7. Donald, B., Claire Kohn, Elizabeth M Booth, Sung-Yun Kang, Kit L Pai, Giorgia Shaw, Myriam Santilli, Karen F Armant, Uimook Buckland, Suk See Choi, J. De Ravin, Morna, Y. Dorsey, Caroline, Kuo, Diego Leon- Rico, Christine Rivat, Natalia Izotova, Kimberly Gilmour, Katie Snell, Jinhua Xu-Bayford Dip, Jinan Darwish, Emma C., Dayna Morris, Leo D Terrazas, Christopher A Wang, Tobias Bauser, B. Paprotka, Douglas, John Kuhns Gregg, E. Hayley, Raymond, John K Everett, Geraldine Honnet, Luca Biasco, Peter E Newburger, Frederic D., Manuel Bushman, H Bobby Grez, David A Gaspar, and Williams, Harry L Malech, Anne Galy, Adrian J Thrasher, Net4CGD consortium. 2020. Lentiviral gene therapy for X-linked chronic granulomatous disease. Nature Medicine 26: 200-206. DOI: 10.1038/s41591-019-0735-5.

8. Maren Weisser, Uta M., Stefan Demel, Linping Stein, Fabien Chen-Wichmann, Giorgia Touzot, Stefanie Santilli, Christian Sujer, Ulrich Brendel, Marina Siler, Adrian J. Cavazzana, Janine Thrasher, A. G. Reichenbach, Marieke, and Essers, Joachim Schwäble, and Manuel Grez. 2016. Hyperinflammation in patients with chronic granulomatous disease leads to impairment of hematopoietic stem cell functions. Journal of Allergy and Clinical Immunology 138: 219-228. DOI: 10.1016/j.jaci.2015.11.028.

9. Hideaki Nakamura, Jun, Tomoyuki Fang, Hiroyuki Mizukami, Nunoi, and Hiroshi Maeda. 2012. PEGylated Damino acid oxidase restores bactericidal activity of neutrophils in chronic granulomatous disease via hypochlorite. Experimental Biology and Medicine 237: 703-708. https://doi.org/10.1258/ebm.2012.011360.

10. Gianfranco Pasut \& Samuel Zalipsky Edit. 2020. Polymer-Protein Conjugates - From PEGylation and Beyond. 1-485. Amsterdam: Elsevier B.V.

11. Hiroshi Maeda. 2013. The link between infection and cancer: Tumor vasculature, free radicals, and drug delivery to tumors via the EPR effect. Cancer Sci 104: 779-789. DOI:10.1111/cas.12152.

12. Daiki Endo, Kenta, Rika Fujimoto, Hiroko Hirose, Mizuki Yamanaka, Ken-ichi Homme, Noriko Ishibashi, Naohito Miura, and Ohno, and Yasuaki Aratani. 2017. Genetic phagocyte NADPH oxidase deficiency enhances nonviable Candida albicans-induced inflammation in mouse lungs. Inflammation 40: 123-135. DOI:10.1007/s10753-016-0461-9.

13. Jonathan, D., David A. Pollock, A. C. Williams, Mary, Ling Lin Gifford, Xunxiang Li, Jason Du, Stuart H. Fisherman, M. Orkin, Claire, Doerschuk, and Mary C. Dinauer. 1995. Mouse model of X-linked chronic 
granulomatous disease, an inherited defect in phagocyte superoxide production. Nature Genetics 9: 202-209. DOI:10.1038/ng0295-202.

14. Shunsuke Hida, Noriko N.Miura, and Yoshiyuki Adachi, Naohito Ohno. 2005. Effect of Candida albicans cell wall glucan as adjuvant for induction of autoimmune arthritis in mice. Journal of Autoimmunity 25: 93-101. DOI:10.1016/j.jaut.2005.06.002.

15. Jun Fang, Dawei, Hideaki Deng, Teruo Nakamura, Haibo Akuta, Arun K Qin, Khaled lyer, and Hiroshi Maeda Greish. 2008. Oxystress inducing antitumor therapeutics via tumor-targeted delivery of PEG-conjugated Damino acid oxidase. International Journal of Cancer 122: 1135-1144. DOI:10.1002/ijc.22982.

16. Morgenstern, David E., A. C. Mary, Ling Lin Gifford, Claire M. Li, Doerschuk, and Mary C. Dinauer. 1997. Absence of respiratory burst in X-linked chronic granulomatous disease mice leads to abnormalities in both host defense and inflammatory response to Aspergillus fumigatus. The Journal of Experimental Medicine 185: 207-218. DOI:10.1084/jem.185.2.207.

17. Mg Schäppi, Christine, L. Deffert, Fr Fiette, Gaëtan Gavazzi, Dc Herrmann, and Karl-Heinz Krause Belli. 2008. Branched fungal $\beta$-glucan causes hyperinflammation and necrosis in phagocyte NADPH oxidase-deficient mice. The Journal of Pathology 214: 434-444. DOI: 10.1002/path.2298.

18. Brahm, H., Wei Segal, Jennifer J. Han, Myungsoo Bushey, Zahida Joo, Joy Bhatti, Carly G. Feminella, R. Dennis, Fiona E. Robert Vethanayagam, Maegan Yull, Paul K. Capitano, Hans Wallace, John W. Minderman, Michael B. Christman, Jefferson Sporn, Donald C. Chan, Steven M. Vin, Luigina R. Holland, Sarah L. Romani, Michael L. Gaffen, Freeman, and Timothy S. Blackwell. 2010. NADPH oxidase limits innate immune responses in the lungs in mice. PLoS One 5: e9631.

19. https://doi.org/10.1371/journal.pone.0009631.

20. Song, Z., G. Huang, L. Chiquetto Paracatu, D. Grimes, J. Gu, C. J. Luke, R. A. Clemens, and M. C. Dinauer. 2020. NADPH oxidase controls pulmonary neutrophil infiltration in the response to fungal cell walls by limiting LTB4. Blood 135: 891-903. DOl:10.1182/blood.2019003525.

21. Mark, T., Quinn, and Igor A. Schepetkin. 2009. Role of NADPH oxidase in formation and function of multinucleated giant cells. Journal of Innate Immunity 1: 509-526. doi:10.1159/000228158.

22. Setoguchi, K., M. Takeya, T. Akaike, M. Suga, R. Hattori, H. Maeda, M. Ando, and K. Takahashi. 1996. Expression of inducible nitric oxide synthase and its involvement in pulmonary granulomatous inflammation in rats. The American Journal of Pathology 149: 2005-2022. https://doi.org/10.1164/ajrccm.156.1.9609140.

\section{Figures}




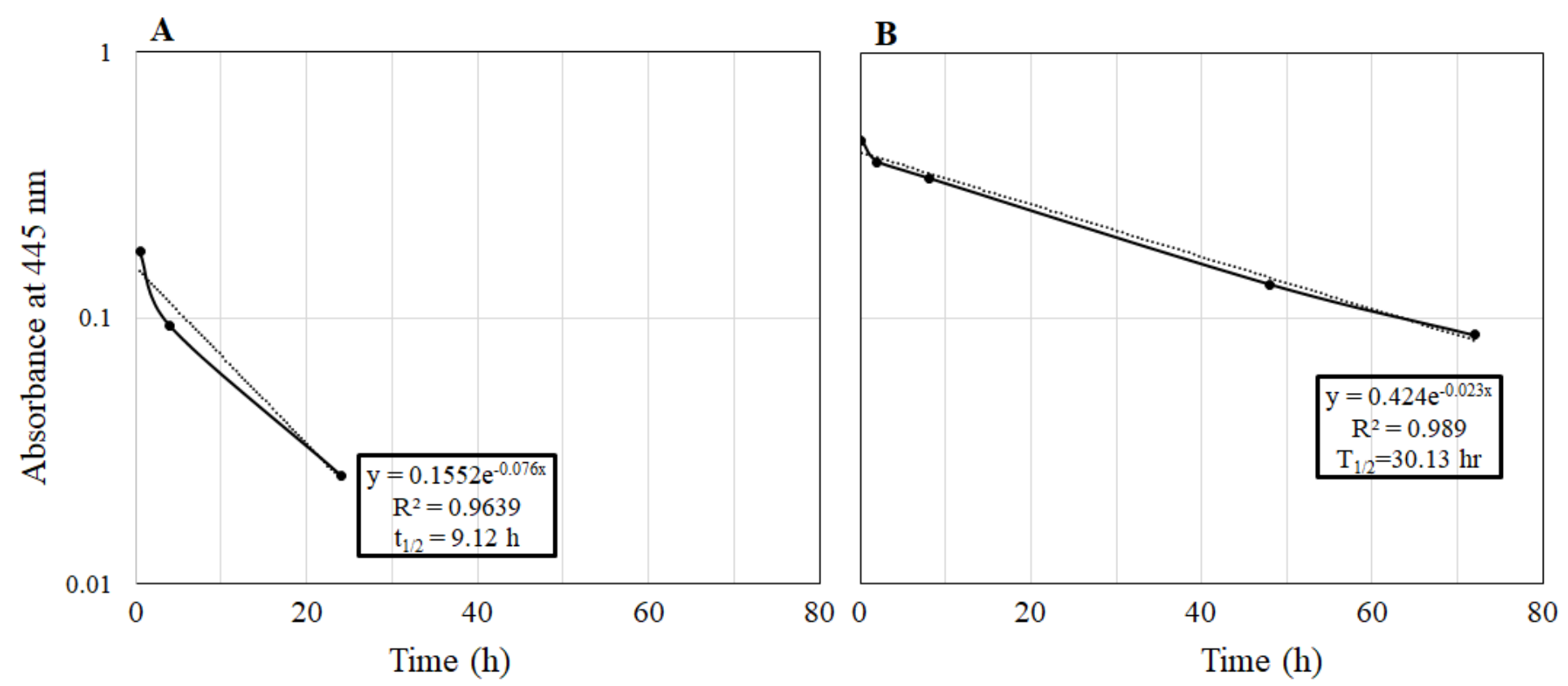

Figure 1

In vivo pharmacokinetics of fDAO and PEG-fDAO by enzymatic activities using the plasma. The $y$-axis shows the log of the absorbance at $445 \mathrm{~nm}$, which denotes the production of pyruvic acid from D-alanine by D-amino acid oxidase. In this figure, two typical sets of exponential curve fitting analysis along with the mathematical formula are shown for fDAO (Figure 1A) and PEG-fDAO (Figure 1B) (Microsoft ExcelR). The mathematical calculation for biological half-life $(x=t 1 / 2)$ is also shown in the figure. $R 2$ is $R$ squared, which is coefficient of determination that is predictable from the independent variable 

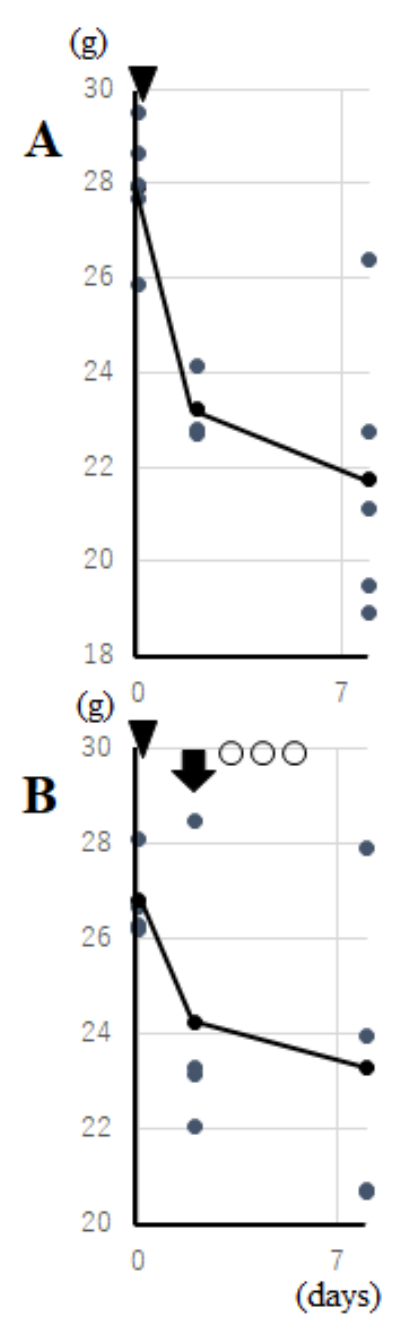

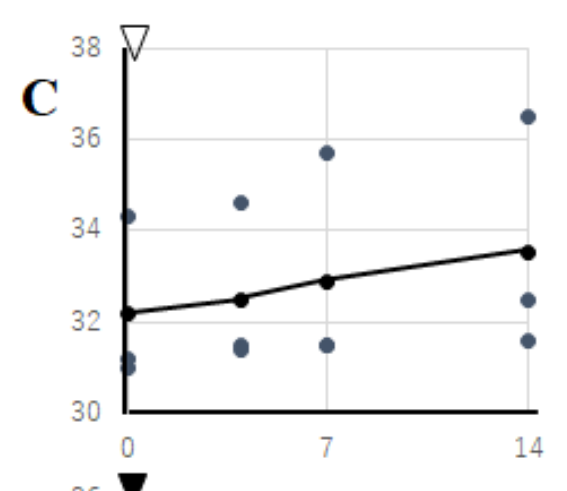

D
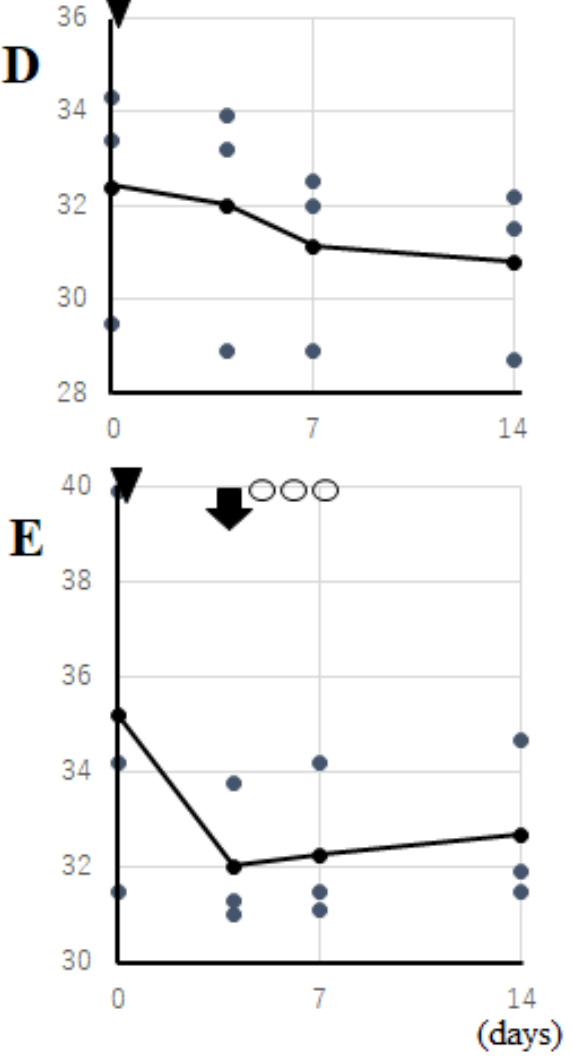
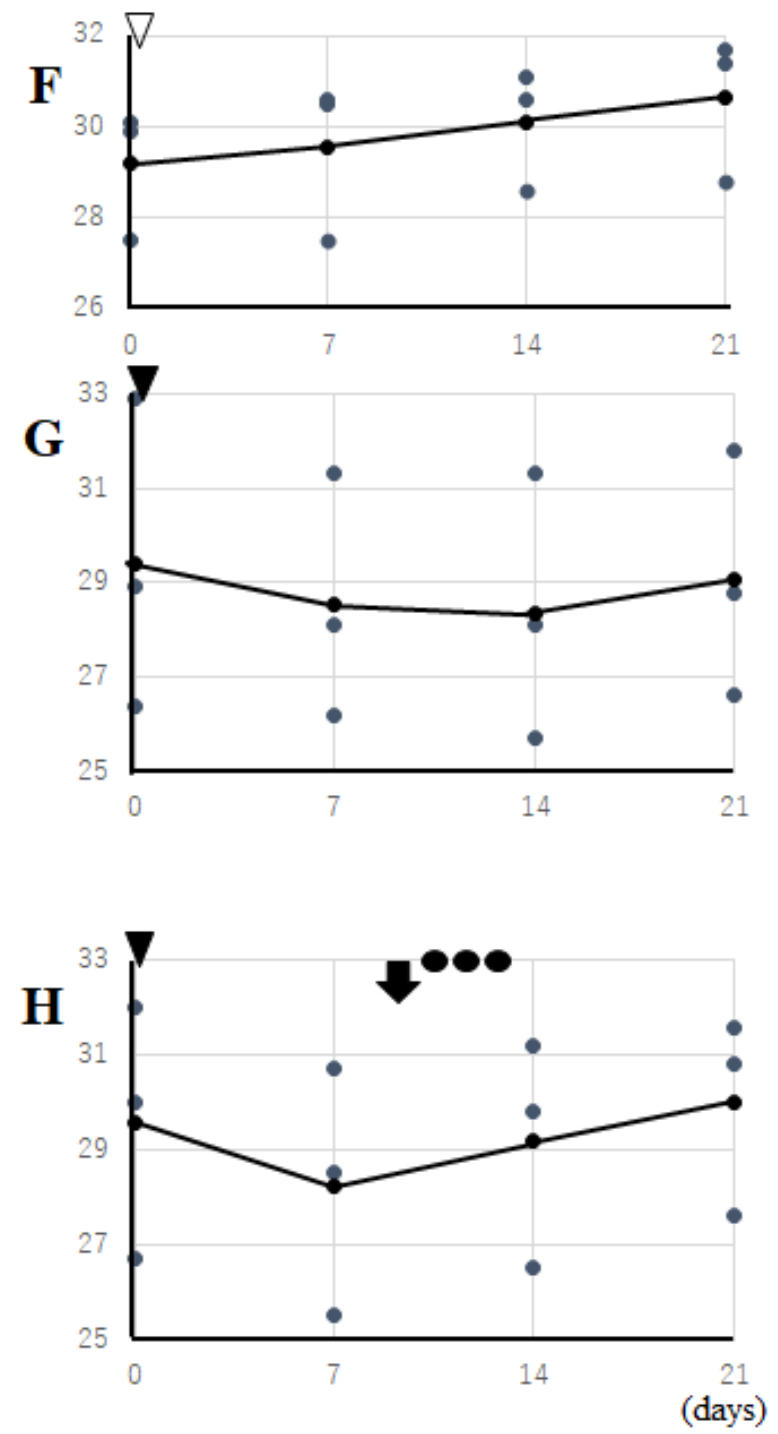

Figure 2

Changes in body weight in each experiment. CGD mice were challenged with intranasal administration of $107 \mathrm{nCA}$ cells (囚) in a volume of $30 \mu \mathrm{L}$ of PBS, and control mice were administered $30 \mu \mathrm{L}$ of PBS alone (囚). PEG-fDAO $(\Downarrow)$ was intravenously administered on the second day (Figure 2B), fourth day (Figure 2E), and ninth day (Figure 2H),

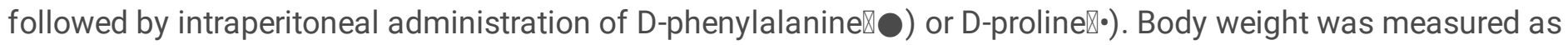
indicated. The small dots show the body weight in the course of each experiment. The solid line indicates the mean body weight. Since the age of the mice at the start of each experiment was not exactly the same, the average body weight at the start of the experiment differed across the experiments. 

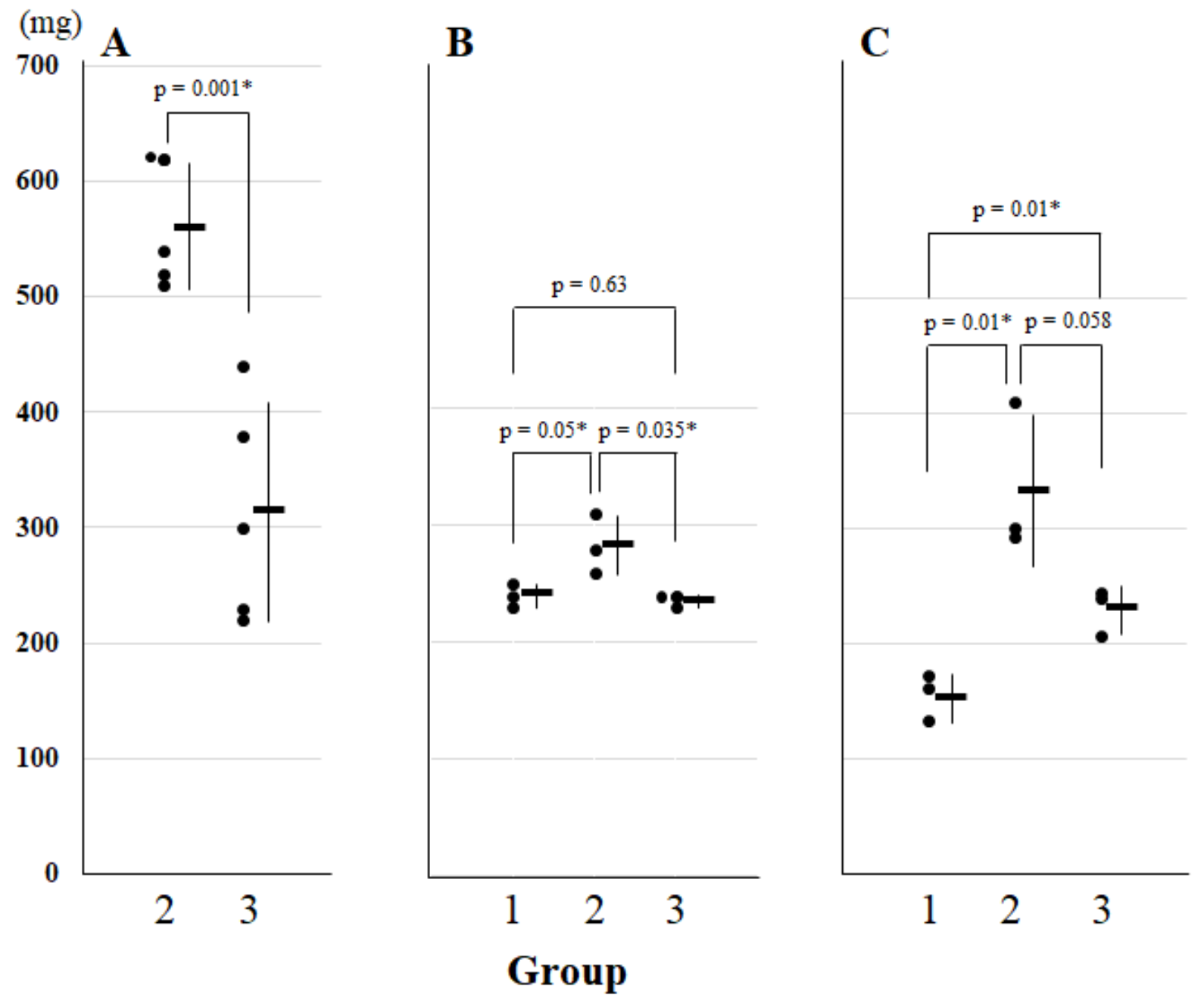

Figure 3

Change in the lung weight in each experiment. The small dots $(\cdot)$ show the lung weight in Exp-1/-2/-3 (Figure 3A/B/C). The control, nCA and nCA+PEG-fDAO group were indicated as 1, 2, and 3. Solid bars ( - ) and the vertical bars indicate mean and \pm 2 SD of the lung weight $(\mathrm{mg})$. An asterisk $\left({ }^{*}\right)$ indicates a significant difference between the groups in each experiment. 
A

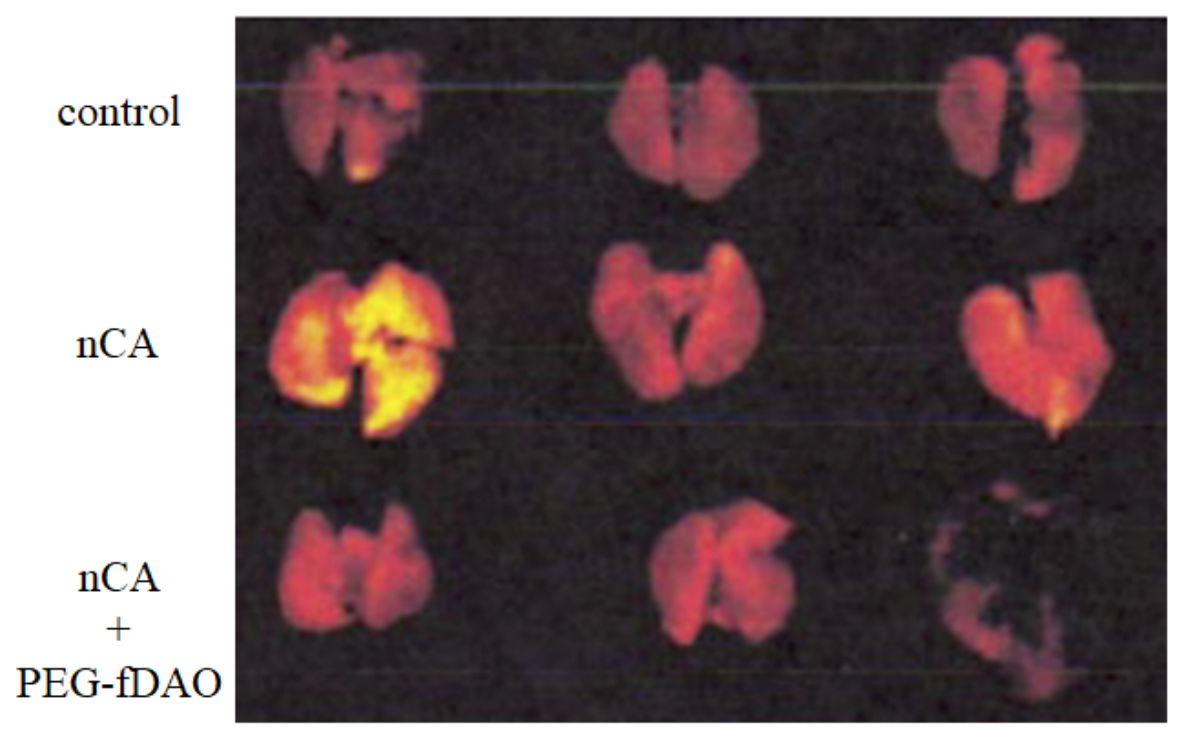

$\operatorname{Exp} 2$
B

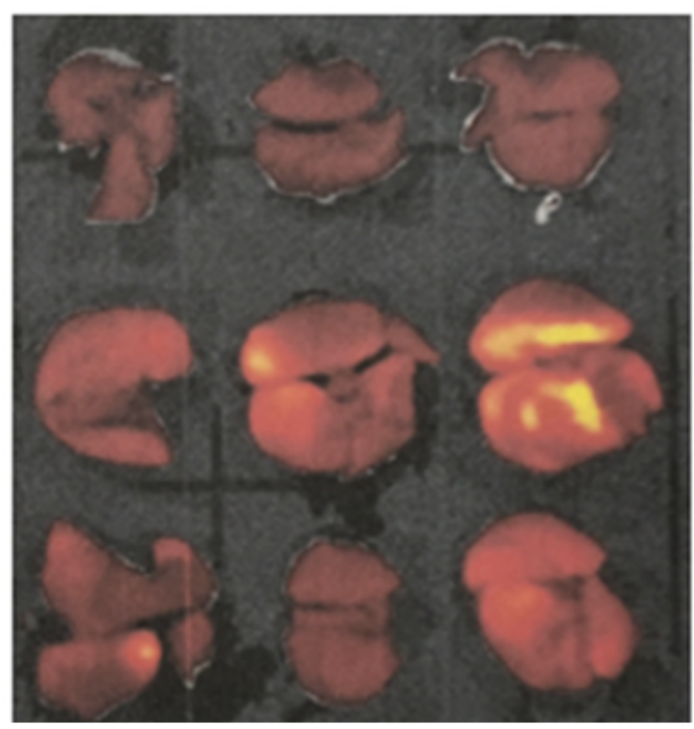

$\operatorname{Exp} 3$

\section{Figure 4}

Fluorescence imaging of the lung tissues during each experiment. The lung tissues of mice from the control, nCA, and nCA+PEG-fDAO groups in Exp-2 (A) and Exp-3 (B) are shown.
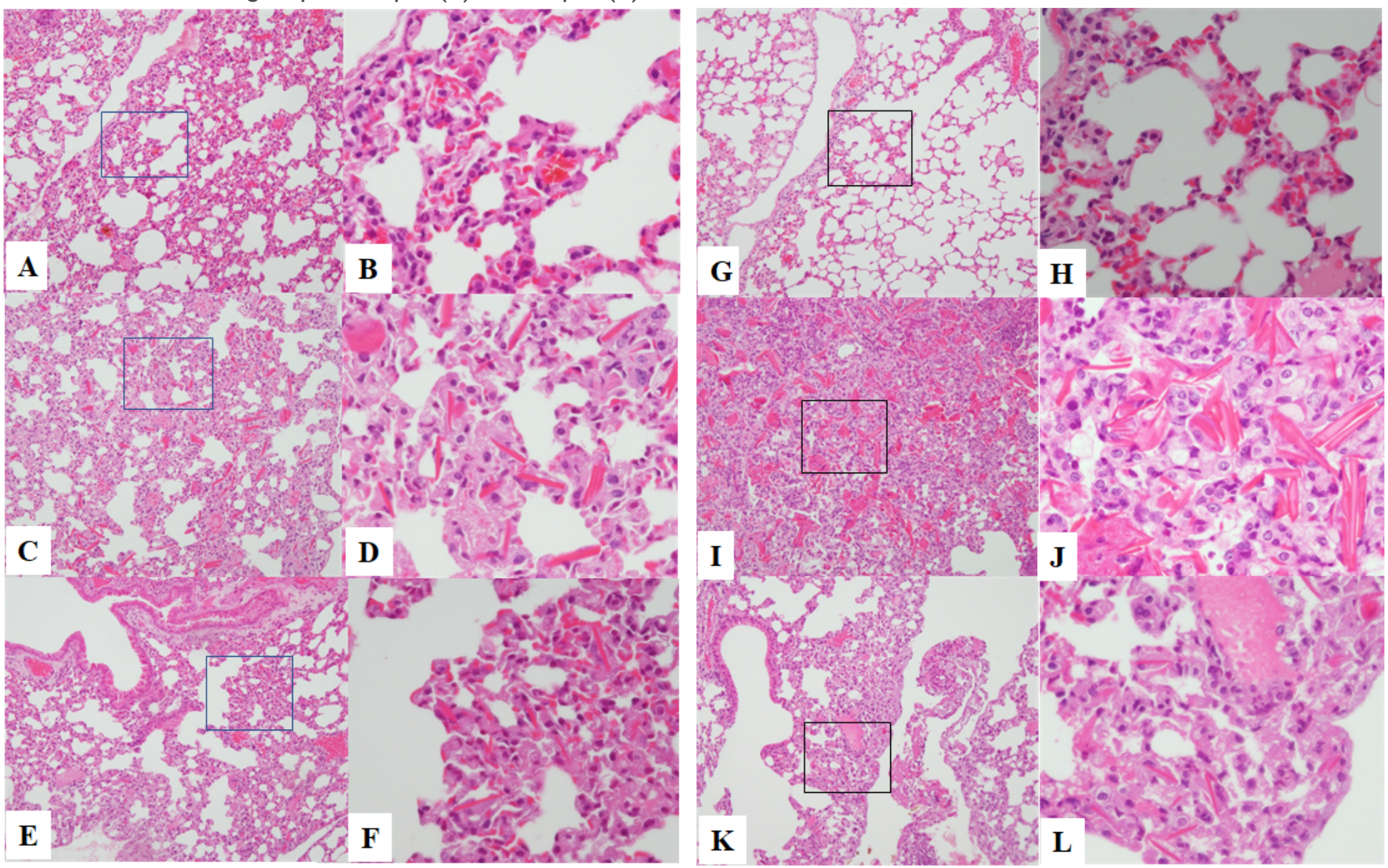

Figure 5 
Pathological findings of the lung tissues in Exp-2 and Exp-3. Lung pathologies in Exp-2 (Figure 5A-F) and Exp-3 (Figure 5G-L) are shown, where $\mathrm{A} / \mathrm{B}$ and $\mathrm{G} / \mathrm{H}, \mathrm{C} / \mathrm{D}$ and $\mathrm{I} / \mathrm{J}, \mathrm{E} / \mathrm{F}$ and $\mathrm{K} / \mathrm{L}$ indicate the control, $\mathrm{nCA}$ and PEG-fDAO group, respectively. $A / C / E / G / I / K$ and $B / D / F / H / J / L$ show original magnifications of $\times 100$ and $\times 400$, respectively. The boxed areas are amplified in the right panels. The nCA group showed pneumonia with many eosinophilic filaments and neutrophils phagocytizing the filaments in Exp-2 (Figure 5C/D) and Exp-3 (Figure5I/J). In addition, a somewhat granulomatous appearance showing a mixed accumulation of neutrophils, macrophages, and lymphoid cells was noted in Exp-3 (Figure 5l/J). The inflammatory changes were milder in the PEG-fDAO group (Figure $5 E / F / K / L$ ) than in the nCA group (Figure $5 \mathrm{C} / \mathrm{D} / \mathrm{I} / \mathrm{J}$ ), showing thin eosinophilic filament-phagocytizing neutrophils in both Exp-2 and Exp-3.

\section{Supplementary Files}

This is a list of supplementary files associated with this preprint. Click to download.

- SupplementalDatalnflammation.pdf 